\title{
Cost-Consequence Analysis of Using Cangrelor in High Angiographic Risk Percutaneous Coronary Intervention Patients: A US Hospital Perspective
}

\author{
Ivar S. Jensen ${ }^{1}$ (1) - Elizabeth Wu ${ }^{1}$. Philip L. Cyr ${ }^{1,2} \cdot$ Marc Claussen $^{3} \cdot$ Thomas Winkler $^{3} \cdot$ Khalid Salahuddin $^{3}$. \\ Jayne Prats ${ }^{4} \cdot$ Kenneth W. Mahaffey ${ }^{5}$. Charles Michael Gibson ${ }^{6} \cdot$ Philippe Gabriel Steg $^{7} \cdot$ Gregg W. Stone $^{8}$. \\ Deepak L. Bhatt ${ }^{9}$
}

Accepted: 7 July 2021 / Published online: 31 July 2021

(c) The Author(s) 2021

\begin{abstract}
Objectives The objective of this study was to evaluate a US hospital's cost implications and outcomes of cangrelor use in percutaneous coronary intervention (PCI) patients with two or more angiographic high-risk features (HRFs), including avoidance of oral $\mathrm{P} 2 \mathrm{Y}_{12}$ inhibitor pretreatment in patients requiring cardiac surgery. Intravenous cangrelor provides direct, immediate onset and rapid-offset $\mathrm{P} 2 \mathrm{Y}_{12}$ inhibition, which may reduce the necessity for oral $\mathrm{P} 2 \mathrm{Y}_{12}$ pretreatment.

Methods A decision analytic model was developed, estimating the annual impact over 3 years of cangrelor availability. Ischemic and bleeding events ( $48 \mathrm{~h}$ ) from randomized clinical trial data were extrapolated to 30 days. Event costs were from the CHAMPION PHOENIX Economics substudy. Rates of coronary artery disease (CAD) presentation, PCI, oral P2 $Y_{12}$ pretreatment, and inpatient hospitalization costs were from published literature and clinical experts. Scenario analyses evaluated the impact of cangrelor availability on potential reduced $\mathrm{P}_{2} \mathrm{Y}_{12}$ pretreatment rates by $50-100 \%$. Drug costs were 2019 wholesale acquisition costs and, where necessary, all costs were adjusted to 2019 dollars.

Results In a hospital treating 1000 CAD PCI inpatients annually, increasing cangrelor use from 11 to $32 \%$ resulted in a reduction in 48 -h ischemic events/year by $5.7 \%$, while bleeding events increased by $2.9 \%$. Total costs of $\$ 1,135,472$ declined $12.8 \%$, with a $50 \%$ reduction in $\mathrm{P}_{2} \mathrm{Y}_{12}$ pretreatment or $30 \%$ with no pretreatment. Savings were driven by a decrease in ischemic events, decrease in glycoprotein IIb/IIIa inhibitor use, and less need for and shorter oral $\mathrm{P} 2 \mathrm{Y}_{12}$ inhibitor washout period for surgery patients.

Conclusion Use of cangrelor in patients with two or more angiographic HRFs may improve outcomes and lower hospital budgets, mainly from avoiding surgery delays necessitated by oral $\mathrm{P} 2 \mathrm{Y}_{12}$ inhibitor pretreatment.
\end{abstract}

Ivar S. Jensen

Ivar.Jensen@precisionvh.com

Extended author information available on the last page of the article

\section{Key Points}

In patients with two or more angiographic high-risk features undergoing coronary revascularization, intravenous cangrelor provides potential cost savings at the hospital level by reducing periprocedural ischemic events while lowering the costs from delays in coronary artery bypass graft due to oral $\mathrm{P} 2 \mathrm{Y}_{12}$ inhibitor pretreatment.

Given these findings, as well as the lack of randomized data supporting $\mathrm{P} 2 \mathrm{Y}_{12}$ inhibitor pretreatment in patients undergoing percutaneous coronary intervention (PCI), consideration should be given to the use of cangrelor during PCI in patients with high clinical or angiographic risk. 


\section{Introduction}

Clinical guidelines recommend combining anticoagulants with aspirin plus an oral $\mathrm{P}_{2} \mathrm{Y}_{12}$ inhibitor with or without glycoprotein IIb/IIIa inhibitors (GPI) during percutaneous coronary intervention (PCI) to reduce periprocedural ischemic events [1, 2]. However, oral P2 $\mathrm{Y}_{12}$ inhibitors may exhibit slow onset of platelet inhibition and low response rates, especially among patients with acute coronary syndrome and ST-segment elevation myocardial infarction (STEMI) [3-5]. Delayed response may be due to high baseline platelet reactivity, reduced bioavailability of oral agents in STEMI patients, and delayed metabolism of thienopyridines into their active metabolites [5]. These issues are exacerbated with use of morphine or fentanyl $[6,7]$, which are often coadministered for chest pain and anxiety [3-5]. Consequently, prescribing information for oral $\mathrm{P}_{2} \mathrm{Y}_{12}$ inhibitors includes this risk of delayed and decreased absorption with concomitant opioid administration [8-10]. Recent guidance recommends against routine $\mathrm{P}_{2} \mathrm{Y}_{12}$ pretreatment of NSTEMI patients until the coronary anatomy is known. [1]. Patients may also have risk associated with lesion complexity, further underscoring the need for potent and prompt platelet inhibition. Immediate $\mathrm{P}_{2} \mathrm{Y}_{12}$ inhibition is important [3] but may not be achievable given mean PCI durations of $<20 \mathrm{~min}$ and rapid door-to-first-device times in STEMI [11].

Cangrelor, a novel, intravenous platelet $\mathrm{P}_{2} \mathrm{Y}_{12}$ receptor inhibitor, provides direct, immediate onset and rapid-offset P2 $\mathrm{Y}_{12}$ inhibition for PCI. The safety and efficacy of cangrelor was evaluated in three trials: CHAMPION PHOENIX, CHAMPION PCI, and CHAMPION PLATFORM [11-13]. The significant reduction in ischemic events (major adverse cardiovascular event [MACE]: death, myocardial infarction (MI), ischemia-driven revascularization [IDR], or stent thrombosis [ST]) at $48 \mathrm{~h}$ versus clopidogrel in CHAMPION PHOENIX led to the approval of cangrelor. A pooled analysis of the CHAMPION trials confirmed that cangrelor was effective in reducing MACE [14]. Despite its demonstrated efficacy in an all-comers PCI population in CHAMPION PHOENIX, which was consistent in the US and non-US subgroups [15] and is included in recent clinical guidelines $[1,16,17]$, in practice cangrelor may be restricted to subsets of PCI patients, such as those with high acuity presentation (STEMI, NSTEMI) and those who are unable to take oral antiplatelets. This limited use of cangrelor stems in part from its perceived impact on hospital budgets due to its acquisition cost compared with oral $\mathrm{P} 2 \mathrm{Y}_{12}$ inhibitors.

An analysis from CHAMPION PHOENIX showed that angiographic high-risk features (HRFs) are a powerful predictor of 48-h MACE [18]. Angiographic HRFs include long lesions ( $>20 \mathrm{~mm}$ ), bifurcation (diameter of stenosis $\geq 50 \%$ ), eccentric anatomy, tortuous (moderate/ severe), angulated (moderate/severe), calcified (moderate/ severe), left main (diameter of stenosis $\geq 50 \%$ ), thrombotic lesions, or multi-lesion PCI [18]. Notably, a majority (56\%) of patients presenting with stable angina undergoing PCI had two or more angiographic HRFs [18]. An analysis of CHAMPION PHOENIX evaluating timing of 48-h MACE found the vast majority of events, regardless of patient presentation, occurred within $2 \mathrm{~h}$ following randomization [19-21]. Thus, utilizing a potent, rapid-acting $\mathrm{P}_{2} \mathrm{Y}_{12}$ inhibitor during and immediately after PCI to reduce the risk of periprocedural ischemic events seems warranted [22]. Given cangrelor's immediate $(<2 \mathrm{~min})$ onset of action and its rapid ( $1 \mathrm{~h}$ post-discontinuation) offset, hospitals could decrease costs with cangrelor use by reducing the proportion of inpatients pretreated with oral $\mathrm{P} 2 \mathrm{Y}_{12}$ inhibitors and thus potentially avoid prolonged hospitalization in patients who require delays to coronary artery bypass graft (CABG) surgery to allow for oral $\mathrm{P} 2 \mathrm{Y}_{12}$ inhibitor washout.

The objective of the present study was to model the cost implications and outcomes from the perspective of a US hospital of using cangrelor in PCI patients with increasing numbers of angiographic HRFs, including the economic benefit of reducing oral $\mathrm{P} 2 \mathrm{Y}_{12}$ inhibitor pretreatment and the subsequent delay to $\mathrm{CABG}$ in patients requiring surgery. The model was developed using Microsoft Excel 2016 software (Microsoft Corporation, Redmond, WA, USA).

\section{Materials and Methods}

A decision analytic model was developed using Microsoft Excel (Fig. 1) to estimate annual costs and outcomes of treating an increasing number of angiographic HRF PCI patients with cangrelor and direct economic benefit from reduction in MACE, as well as the indirect economic benefit of reducing pretreatment with oral $\mathrm{P}_{2} \mathrm{Y}_{12}$ inhibitors, per the 2020 European Society of Cardiology (ESC) guidelines [1, 2]. The model adopted a 3-year time horizon, from the perspective of a US hospital, for coronary artery disease (CAD) patients requiring PCI or going directly to CABG. PCI patients were further stratified into four subgroups: those with none, one, two, or three or more angiographic HRFs. Since the angiographic HRFs are not known until after the angiogram, these levels were considered a reasonable proxy to represent the level of clinical risk in a patient about to undergo PCI. A similar proportion of patients across HRF subgroups were presumed to be pretreated before PCI with oral $\mathrm{P} 2 \mathrm{Y}_{12}$ inhibitors. Periprocedural antiplatelet options were either cangrelor alone or clopidogrel with or without planned GPI (designated GPI use before PCI). It was assumed that a proportion of CABG patients pretreated 


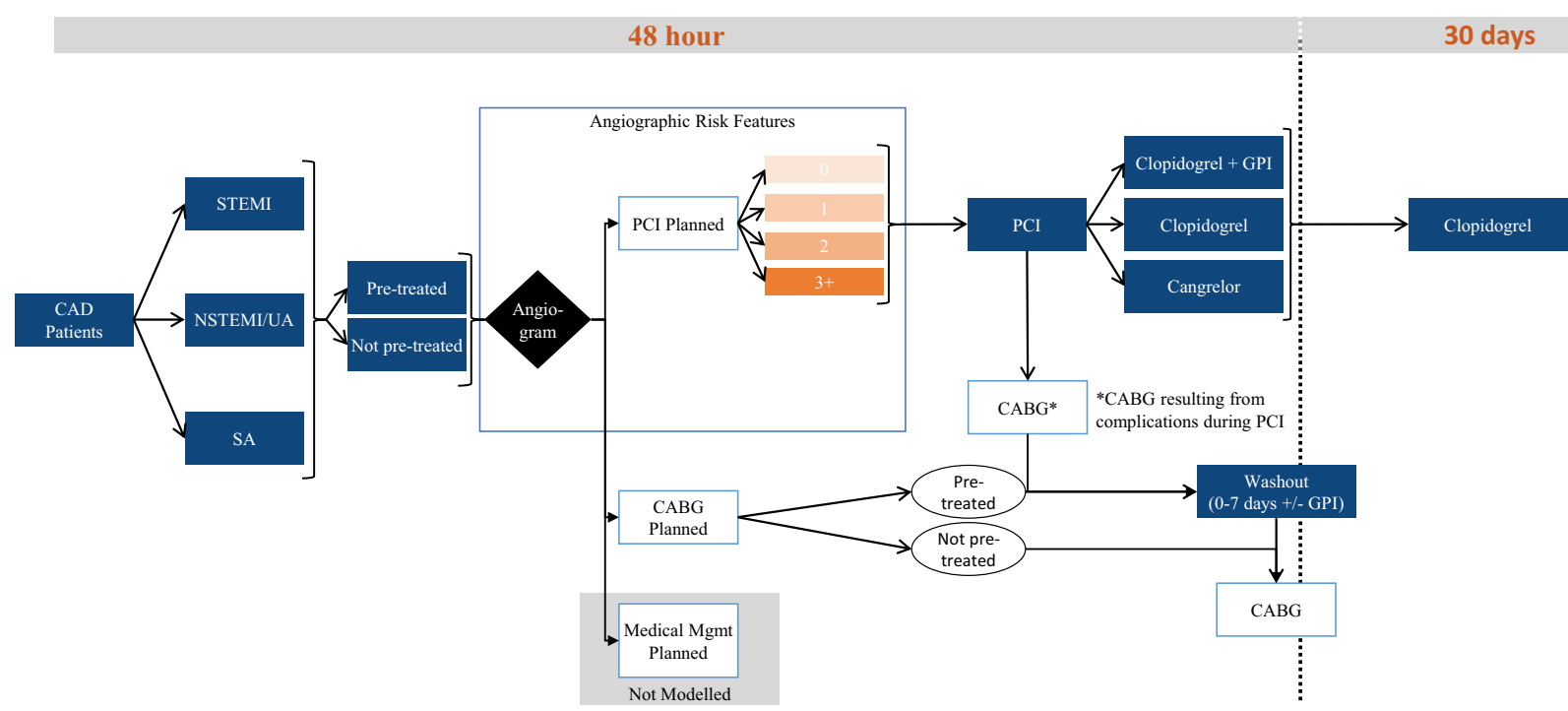

Fig. 1 Decision analytic model structure. $C A B G$ coronary artery bypass graft, $C H D$ coronary heart disease, GPI glycoprotein IIb/IIIa inhibitors, NSTEMI non-ST segment elevation myocardial infarction,

with an oral $\mathrm{P} 2 \mathrm{Y}_{12}$ inhibitor would also need antiplatelet or anticoagulant bridging therapy during the $\mathrm{P}_{2} \mathrm{Y}_{12}$ inhibitor washout period before surgery. We estimated outcomes and costs during the index admissions through 30 days for an annual population of CAD patients. Ethics approval was not required for this study since the analysis uses only published data from clinical trials.

\section{Model Inputs}

\subsection{Target Population}

Based on data from the Premier Hospital Database of inpatient charges, the proportion of CAD patients by diagnosis was $6 \%, 17 \%$, and $77 \%$ for STEMI-ACS, NSTEMI/UA-ACS, and stable angina, respectively (Online Resource Table 1). Following angiography, $57 \%$ of patients had PCI and 5\% went directly to CABG surgery. Of the patients designated for PCI, $99.8 \%$ received $\mathrm{PCI}$ and $0.2 \%$ received CABG surgery due to a complication during PCI [23]. In CHAMPION PHOENIX, among PCI patients, $17 \%, 32 \%, 27 \%$, and $25 \%$ had none, one, two, or three or more angiographic HRFs, respectively. According to the literature, $33 \%$ of these patients received an oral $\mathrm{P}_{2} \mathrm{Y}_{12}$ inhibitor before PCI [24]. Due to its rapid onset of action, having cangrelor available could reduce the need to pretreat CAD patients. We simulated two scenarios where oral $\mathrm{P}_{2} \mathrm{Y}_{12}$ inhibitor pretreatment was reduced by $50 \%$ and $100 \%$, respectively.
$P C I$ percutaneous coronary intervention, $S A$ stable angina, STEMI ST segment elevation myocardial infarction, $U A$ unstable angina

\subsection{Utilization}

Assumptions for base case and scenario analyses on the utilization of antiplatelet agents, illustrated in Table 1, were informed by clinical experts from the CHAMPION Executive Committee. We assumed low-risk patients (e.g. those with fewer than one angiographic HRF) were only treated with clopidogrel \pm planned GPI, and high-risk patients (e.g. those with two or more angiographic HRFs) might be administered cangrelor. This model assumed an increasing proportion of high-risk patients received cangrelor over 3 years.

\subsection{Ischemic Outcomes}

Forty-eight-hour MACE rates by the subgroups of angiographic HRFs for cangrelor and clopidogrel patients were estimated from the literature [18]. According to Vaduganathan et al., the relative increase in 48-h ischemic events for patients treated with clopidogrel + planned GPI, compared with those treated with cangrelor, was $26.9 \%$ [25]. With this assumption, 48-h MACE for clopidogrel + planned GPI patients were extrapolated within each subgroup by applying the $26.9 \%$ increase relative to the rate for cangrelor patients. The stratification of 48-h MACE rates into individual MACE events for cangrelor, clopidogrel, and clopidogrel + planned GPI patients was based on an analysis by Cavender et al. [19-21]. The proportions were then multiplied by the 48-h MACE rates to obtain the individual ischemic events within subgroups and by treatment. 
Table 1 Utilization share

\begin{tabular}{|c|c|c|c|c|}
\hline Risk factor groups & Current utilization (\%) & Year $1(\%)$ & Year $2(\%)$ & Year $3(\%)$ \\
\hline \multicolumn{5}{|l|}{ 0 Angiographic high-risk factors ${ }^{\mathrm{a}}$} \\
\hline Clopidogrel & 90 & 90 & 90 & 90 \\
\hline Clopidogrel + GPI & 10 & 10 & 10 & 10 \\
\hline Cangrelor & 0 & 0 & 0 & 0 \\
\hline \multicolumn{5}{|l|}{1 Angiographic high-risk factor ${ }^{a}$} \\
\hline Clopidogrel & 90 & 90 & 90 & 90 \\
\hline Clopidogrel + GPI & 10 & 10 & 10 & 10 \\
\hline Cangrelor & 0 & 0 & 0 & 0 \\
\hline \multicolumn{5}{|l|}{2 Angiographic high-risk factors ${ }^{a}$} \\
\hline Clopidogrel & 80 & 80 & 60 & 40 \\
\hline Clopidogrel + GPI & 10 & 7 & 5 & 3 \\
\hline Cangrelor & 10 & 13 & 35 & 57 \\
\hline \multicolumn{5}{|l|}{$\geq 3$ Angiographic high-risk factors ${ }^{a}$} \\
\hline Clopidogrel & 60 & 50 & 40 & 30 \\
\hline Clopidogrel + GPI & 6 & 5 & 4 & 3 \\
\hline Cangrelor & 34 & 45 & 56 & 67 \\
\hline \multicolumn{5}{|c|}{ Total cangrelor use (calculated from the model) } \\
\hline $\begin{array}{l}\text { Cangrelor use in angiographic HRFs } \geq 2 \\
\text { patients }\end{array}$ & 22 & 28 & 45 & 62 \\
\hline Overall cangrelor use & 11 & 15 & 23 & 32 \\
\hline Overall planned GPI use & 9 & 8 & 7 & 6 \\
\hline Overall clopidogrel use & 80 & 77 & 70 & 62 \\
\hline
\end{tabular}

GPI glycoprotein IIb/IIIa inhibitors, HRFs high-risk features

${ }^{a}$ Assumptions were informed by clinical expert opinion

Based on the CHAMPION trials, increases in MACE of approximately $37 \%$ and $26 \%$ were observed from 48 -h until 30 days among cangrelor- and clopidogrel-treated patients, respectively. The percentage increase for each treatment was then applied to the individual 48-h event rates estimated earlier to extrapolate the individual 30-day event rates. The individual 30-day ischemic event rates for clopidogrel + planned GPI patients were extrapolated relative to the cangrelor rates by the same approach. Table 2 illustrates both the 48-h and 30-day outcomes for death, MI, IDR, and ST events across angiographic HRF subgroups.

\subsection{Bleeding Events}

The 48-h combined (cangrelor alone and clopidogrel \pm planned GPI) bleeding rates by angiographic HRFs from the CHAMPION PHOENIX trial were taken from the study by Stone et al. [18]. Events for two bleeding scales were reported: severe/moderate Global Strategies for Opening Occluded Coronary Arteries (GUSTO) and major/minor Thrombolysis in Myocardial Infarction (TIMI). The GUSTO bleeding definition uses clinical acuity and impact to categorize patients into severe or life-threatening, moderate, or mild categories, whereas the TIMI bleeding definition is based on laboratory values of hematocrit or hemoglobin after adjusting for blood transfusions to categorize bleeding severity as major, minor, or minimal [26]. Based on the relative ratio of bleeding rates reported in a pooled analysis of the CHAMPION trials (cangrelor versus clopidogrel) and in a propensity-matched analysis of cangrelor versus clopidogrel + planned GPI, the rates for each angiographic HRF subgroup were estimated by antiplatelet treatment (i.e. cangrelor alone, clopidogrel alone, and clopidogrel + planned GPI) [14, 25].

The MATRIX trial used heparin as an anticoagulant and demonstrated an increase of approximately $90 \%$ in Bleeding Academic Research Consortium (BARC 3 and 5) bleeding from day $2(0.7 \%)$ to day $30(1.33 \%)$ post PCI [26]. In CHAMPION PHOENIX, heparin was used as the predominant anticoagulant and therefore it was assumed that the same extent of increase could be used as a proxy for both GUSTO moderate/severe and TIMI major/minor bleeding. Therefore, the $90 \%$ increase was applied to the 48-h GUSTO and TIMI bleeding rates to estimate the corresponding 30-day bleeding rates. Table 2 illustrates both the 48-h and 30-day outcomes for death, MI, IDR, ST, and bleeding events across angiographic HRF subgroups. 
Table 2 48-hour and 30-day ischemic and bleeding event rates

\begin{tabular}{|c|c|c|c|c|c|c|}
\hline \multirow{2}{*}{$\begin{array}{l}\text { Events by no. of angio- } \\
\text { graphic HRFs }\end{array}$} & \multicolumn{3}{|l|}{48 hours } & \multicolumn{3}{|l|}{30 days } \\
\hline & $\begin{array}{l}\text { Cangrelor }[18] \\
(\%)\end{array}$ & $\begin{array}{l}\text { Clopidogrel } \\
\text { [18] }(\%)\end{array}$ & $\begin{array}{l}\text { Clopidogrel + planned } \\
\text { GPI }[25](\%)\end{array}$ & Cangrelor (\%) & Clopidogrel (\%) & $\begin{array}{l}\text { Clopidogrel + } \\
\text { planned GPI } \\
(\%)\end{array}$ \\
\hline \multicolumn{7}{|l|}{ MACE } \\
\hline 0 & 1.8 & 3.3 & 2.3 & 2.5 & 4.1 & 3.1 \\
\hline 1 & 3.8 & 4.4 & 4.8 & 5.2 & 5.5 & 6.6 \\
\hline 2 & 6.0 & 6.9 & 7.6 & 8.2 & 8.7 & 10.4 \\
\hline$\geq 3$ & 6.4 & 8.7 & 8.1 & 8.8 & 10.9 & 11.1 \\
\hline \multicolumn{7}{|l|}{ MI [19-21] } \\
\hline 0 & 0.9 & 1.7 & 1.3 & 1.2 & 2.1 & 1.8 \\
\hline 1 & 1.8 & 2.2 & 2.8 & 2.5 & 2.8 & 3.8 \\
\hline 2 & 2.9 & 3.5 & 4.4 & 3.9 & 4.4 & 6.0 \\
\hline$\geq 3$ & 3.1 & 4.4 & 4.7 & 4.2 & 5.6 & 6.4 \\
\hline \multicolumn{7}{|l|}{ ST [19-21] } \\
\hline 0 & 0.2 & 0.5 & 0.3 & 0.3 & 0.6 & 0.4 \\
\hline 1 & 0.4 & 0.6 & 0.7 & 0.6 & 0.8 & 0.9 \\
\hline 2 & 0.7 & 1.0 & 1.1 & 0.9 & 1.2 & 1.5 \\
\hline$\geq 3$ & 0.7 & 1.2 & 1.1 & 1.0 & 1.5 & 1.6 \\
\hline \multicolumn{7}{|l|}{ IDR [19-21] } \\
\hline 0 & 0.5 & 0.8 & 0.4 & 0.6 & 1.0 & 0.6 \\
\hline 1 & 1.0 & 1.1 & 0.9 & 1.3 & 1.3 & 1.3 \\
\hline 2 & 1.5 & 1.7 & 1.4 & 2.1 & 2.1 & 2.0 \\
\hline$\geq 3$ & 1.6 & 2.1 & 1.5 & 2.2 & 2.6 & 2.1 \\
\hline \multicolumn{7}{|l|}{ Death [19-21] } \\
\hline 0 & 0.3 & 0.4 & 0.2 & 0.4 & 0.5 & 0.3 \\
\hline 1 & 0.6 & 0.5 & 0.4 & 0.8 & 0.6 & 0.6 \\
\hline 2 & 1.0 & 0.8 & 0.7 & 1.3 & 1.0 & 0.9 \\
\hline$\geq 3$ & 1.0 & 1.0 & 0.7 & 1.4 & 1.2 & 1.0 \\
\hline \multicolumn{7}{|c|}{ GUSTO severe/moderate bleeding $[14,18,25,26]$} \\
\hline 0 & 0.11 & 0.08 & 0.21 & 0.21 & 0.16 & 0.40 \\
\hline 1 & 0.11 & 0.08 & 0.21 & 0.21 & 0.16 & 0.40 \\
\hline 2 & 0.11 & 0.08 & 0.21 & 0.21 & 0.16 & 0.40 \\
\hline$\geq 3$ & 0.19 & 0.14 & 0.37 & 0.36 & 0.27 & 0.70 \\
\hline \multicolumn{7}{|c|}{ TIMI major/minor bleeding $[14,18,25,26]$} \\
\hline 0 & 0.04 & 0.03 & 0.13 & 0.07 & 0.05 & 0.26 \\
\hline 1 & 0.04 & 0.03 & 0.13 & 0.07 & 0.05 & 0.26 \\
\hline 2 & 0.04 & 0.03 & 0.13 & 0.07 & 0.05 & 0.26 \\
\hline$\geq 3$ & 0.06 & 0.04 & 0.20 & 0.11 & 0.07 & 0.38 \\
\hline
\end{tabular}

GPI glycoprotein IIb/IIIa inhibitors, GUSTO Global Use of Strategies to Open Occluded Arteries, HRFs high-risk features, IDR ischemia-driven revascularization, $M A C E$ major adverse cardiovascular event, $M I$ myocardial infarction, $S T$ stent thrombosis, TIMI thrombolysis in myocardial infarction

\subsection{Glycoprotein Ilb/IIla Inhibitor Bailout}

GPI bailout is defined as the unplanned use of a GPI after the procedure start. In CHAMPION PHOENIX, the rate of GPI bailout was significantly lower among patients treated with cangrelor compared with clopidogrel [11].

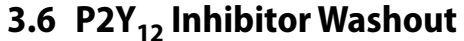

Regardless of presentation, inpatients requiring CABG (either patients going direct to surgery following angiography, or cases during or following the index PCI procedure) who have previously been treated with an oral $\mathrm{P}_{2} \mathrm{Y}_{12}$ 
inhibitor may require 'washing-out' of the antiplatelet effect of these oral agents to reduce the risk of perioperative bleeding. This approach is recommended in practice guidelines as well as the package inserts for each of the oral $\mathrm{P} 2 \mathrm{Y}_{12}$ inhibitors (7-9). An intravenous antiplatelet and/or intravenous anticoagulant may be initiated during the washout period to avoid ischemic events. The proportion of patients by duration of washout for each $\mathrm{P} 2 \mathrm{Y}_{12}$ antiplatelet regimen was based on a Premier Database analysis of inpatient stays (Online Resource Table 3). In this model, it was assumed that clopidogrel + planned GPI has the same duration of washout as clopidogrel alone. In addition, as a simplifying assumption for washout costs, it was assumed patients taking an oral $\mathrm{P}_{2} \mathrm{Y}_{12}$ inhibitor would be switched to an intravenous antiplatelet during washout $[17,27]$. We assumed the GPI would be stopped approximately $4 \mathrm{~h}$ before starting CABG surgery.

\subsection{Economic Inputs}

Ischemic event costs for MI, IDR, and ST were informed by the CHAMPION PHOENIX substudy (Online Resource Table 2). Published inpatient per diem cost was applied to the washout time [28]. Where necessary, all costs were adjusted to 2019 dollars using the medical component of the Bureau of Labor Statistics Consumer Price Index [29].

Potential GPI options for PCI included abciximab, eptifibatide, and tirofiban. The mix of GPI utilization was from the CHAMPION PCI trial. The GPI utilization mix applied in the washout period was adjusted to reflect the practice that only eptifibatide and tirofiban are employed for bridging. Drug costs were based on dosing regimens from the prescribing information of each drug and 2019 wholesale acquisition costs [30]. Table 3 contains the economic inputs used in the model.

\subsection{Sensitivity Analyses}

A deterministic sensitivity analysis (DSA) was performed to systematically examine the impact of each model parameter on the base-case model results. Parameter estimates varied by $\pm 20 \%$, where possible; $100 \%$ was used as the maximum upper range where the $20 \%$ parameter variation could not be applied (i.e. if a parameter estimate was $90 \%$, it would not have been possible to increase that parameter estimate by $18 \%$ [ $20 \%$ of $90 \%$ ], as a percentage cannot exceed $100 \%$ ).

\section{Results}

For a hypothetical hospital treating 1000 inpatient CAD patients, approximately 565 patients were expected to undergo PCI and 50 were expected to undergo CABG
Table 3 Economic inputs

\begin{tabular}{ll}
\hline & Cost \\
\hline Drugs $^{\mathbf{a}}$ [30] & \\
Cangrelor $(50 \mathrm{mg}$ vial) & $\$ 749.00$ \\
Clopidogrel $(75 \mathrm{mg})^{\mathrm{b}}$ & $\$ 0.09$ \\
Abciximab $(10 \mathrm{mg} / 5 \mathrm{~mL})$ & $\$ 1348.18$ \\
Eptifibatide $(0.75 \mathrm{mg} / \mathrm{mL}, 100 \mathrm{~mL}$ vials) & $\$ 270.00$ \\
Tirofiban $(3.75 \mathrm{mg} / 15 \mathrm{~mL}$ vial) & $\$ 222.56$ \\
GPI drug cost per treatment (calculated) & \\
Planned GPI & $\$ 1287$ \\
GPI bailout & $\$ 1750$ \\
GPI use during washout of oral P2Y ${ }_{12}$ inhibitors (per & $\$ 1348$ \\
24 h) & \\
Average cost per event ${ }^{\mathrm{c}}($ Online Resource Table 2) & \\
MI & $\$ 6448$ \\
ST & $\$ 40,379$ \\
IDR & $\$ 23,644$ \\
GUSTO severe/moderate bleeding & $\$ 11,778$ \\
TIMI major/minor bleeding & $\$ 14,135$ \\
Per diem cost & \\
Inpatient [28, 29] & \\
\hline
\end{tabular}

GPI glycoprotein IIb/IIIa inhibitors, GUSTO Global Use of Strategies to Open Occluded Arteries, IDR ischemia-driven revascularization, $M I$ myocardial infarction, ST stent thrombosis, TIMI thrombolysis in myocardial infarction, WAC wholesale acquisition cost

${ }^{\mathrm{a}} \mathrm{WAC}$ cost was based on per vial, bag, or pill

${ }^{\mathrm{b}} \mathrm{A} 300 \mathrm{mg}$ loading dose was used for the clopidogrel regimen

${ }^{\mathrm{c}}$ Cost of severe/major bleeding was used to estimate bleeding treatment costs

${ }^{\mathrm{d}}$ Per diem cost was applied to washout time

surgery each year. The remainder of patients were managed medically and were thus excluded from the cost analysis. Among the patients in whom PCI was planned, 99.8\% ( $n=564)$ received PCI, while $0.2 \%$ (1 patient) received CABG surgery during the index PCI admission (Online Resource Table 1) [23].

In the base case, oral $\mathrm{P} 2 \mathrm{Y}_{12}$ pretreatment was assumed to be $33 \%$ and cangrelor use was $22 \%$ in patients with two or more angiographic HRFs (or $11 \%$ in all PCI patients). In other scenarios, oral $\mathrm{P} 2 \mathrm{Y}_{12}$ pretreatment was assumed to be $17.5 \%$ ( $50 \%$ less than the base case) or $0 \%$ (no pretreatment at all), and cangrelor use in patients with two or more angiographic HRFs increased from $28 \%$ in year 1 to $62 \%$ in year 3 (or $15 \%$ in year 1 to $32 \%$ in year 3 in all PCI patients, while planned GPI use was reduced from $9 \%$ to $6 \%$ over the same time (Table 1).

The total annual number of 48-h MACEs was reduced from the base case of 32.5 to 30.7 by year 3 in the scenario (Fig. 2a). This percentage reduction versus base case was $1.1 \%$ in year 1 and $5.7 \%$ in year 3. A similar trend in reduction was seen for 30-day MACE (Fig. 2b). 


\section{A}

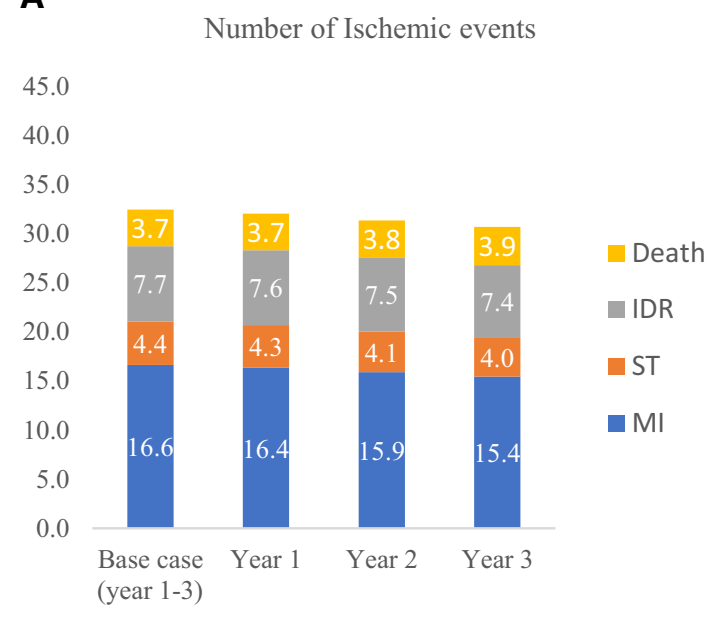

\section{C}

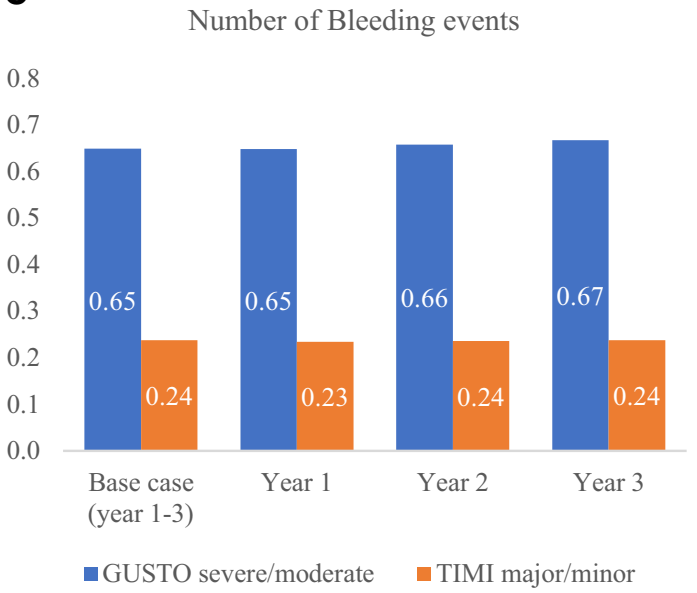

Fig. 2 (A) 48-hour ischemic events; (B) 30-day ischemic events; (C) 48-hour bleeding events; and (D) 30-day bleeding events. GUSTO Global Use of Strategies to Open Occluded Arteries, IDR ischemia-

The total annual number of GUSTO severe/moderate bleeding events was increased from $0.65(48-\mathrm{h})$ and 1.23 (30-day) in the base case to 0.67 (48-h) and 1.27 (30-day) by year 3 in the scenario. However, 48-h and 30-day TIMI major/minor bleeding events were similar for the base case and scenario $(48-\mathrm{h}=0.24,30$-day $=0.45)$ (Fig. 2c, d). Using the GUSTO definition, bleeding event costs for 30-days remained flat (approximately $\$ 14,500-\$ 15,000$ per year, or $<2 \%$ of total costs) over the 3 -year period.

Figure $3 \mathrm{a}, \mathrm{b}$ show the total costs by category with reduced $\mathrm{P} 2 \mathrm{Y}_{12}$ pretreatment (and no pretreatment) and increased cangrelor use over the 3 -year period. The annual costs were projected to decline from $\$ 1,135,472$ in the base case to $\$ 990,533$ ( $\$ 811,823$ with no pretreatment) in year 3 . With ischemic events comprising the majority of total costs $(69.6 \%$ in the base case and $52.6 \%$ by year 3$)$, costs decreased from $\$ 597,647$ in the base case to $\$ 565,040$ in
B
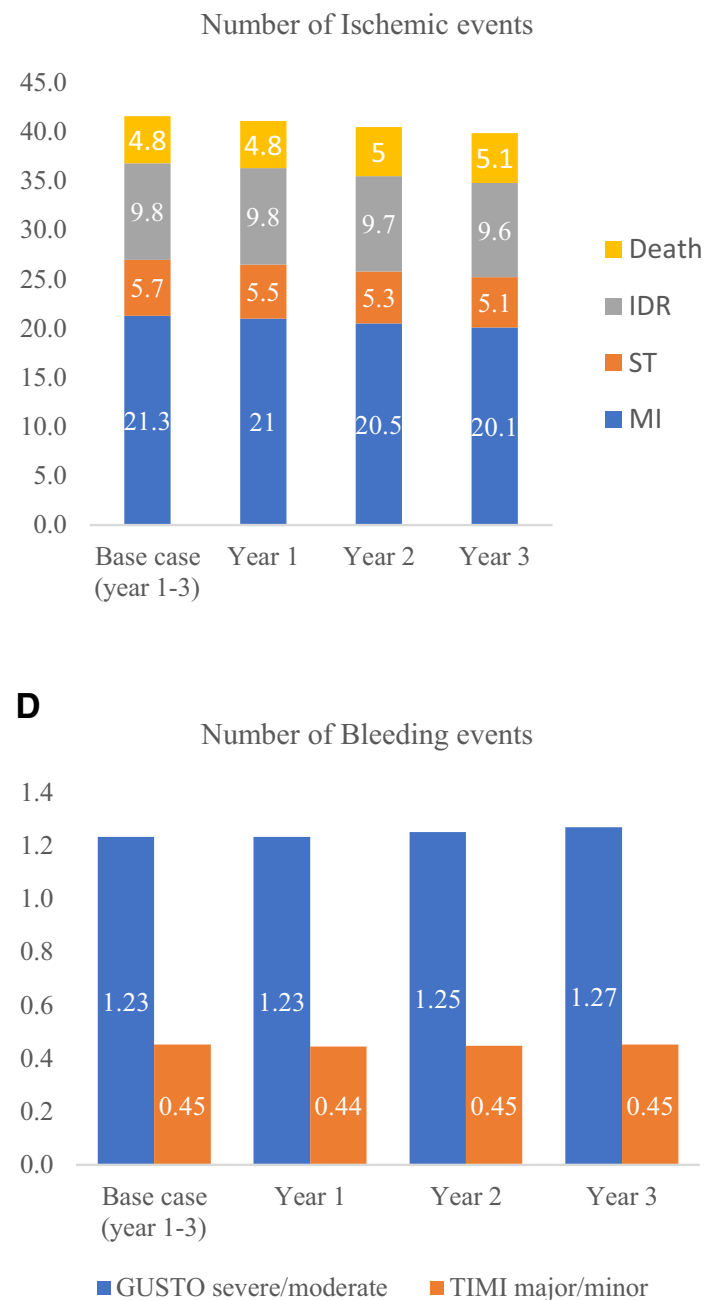

driven revascularization, $M I$ myocardial infarction, $S T$ stent thrombosis, TIMI thrombolysis in myocardial infarction

year 3. A similar trend was observed in GPI bailout cost but at a smaller magnitude. Washout costs declined by $47 \%$, from $\$ 380,752$ in the base case to $\$ 199,934$ ( $\$ 21,224$ with no pretreatment) in the scenario.

Total drug acquisition cost was the second-largest cost driver. Drug cost increased from $\$ 112,844$ in the base case to $\$ 181,677$ by year 3, representing an increase of $61 \%$. The overall budget impact for years 1,2 , and 3 was $-\$ 179,947$, $-\$ 162,883$, and $-\$ 145,819$, respectively, in the case of $50 \%$ reduced $\mathrm{P} 2 \mathrm{Y}_{12}$ pretreatment, and $-\$ 358,278,-\$ 340,964$, and $-\$ 323,649$ for years $1-3$ when no $\mathrm{P}_{2} \mathrm{Y}_{12}$ pretreatment was simulated.

A DSA was performed by varying each point estimate by $\pm 20 \%$, where possible. The DSA demonstrated the model was most susceptible to epidemiology assumptions, including the proportion of patients in whom PCI was planned and the proportion of patients who were pretreated with oral 
A

(Scenario: $50 \%$ reduction in pretreatment)

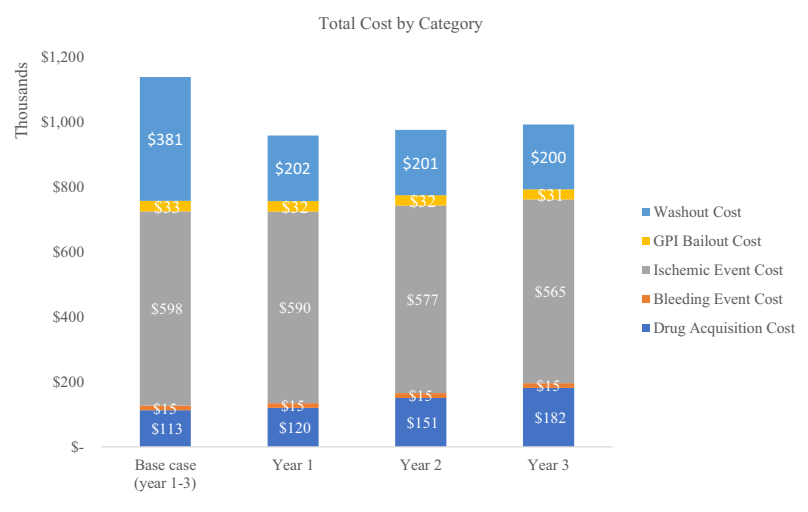

\section{B}

(Scenario: $100 \%$ reduction in pretreatment)

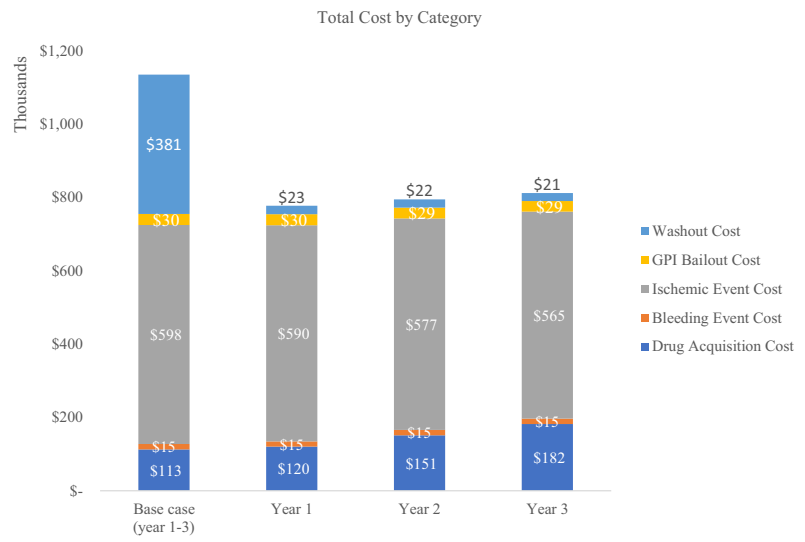

Fig. 3 Total costs and budget impact (A) Scenario: 50\% reduction in pretreatment; (B) Scenario: 100\% reduction in pretreatment. GPI glycoprotein IIb/IIIa inhibitors

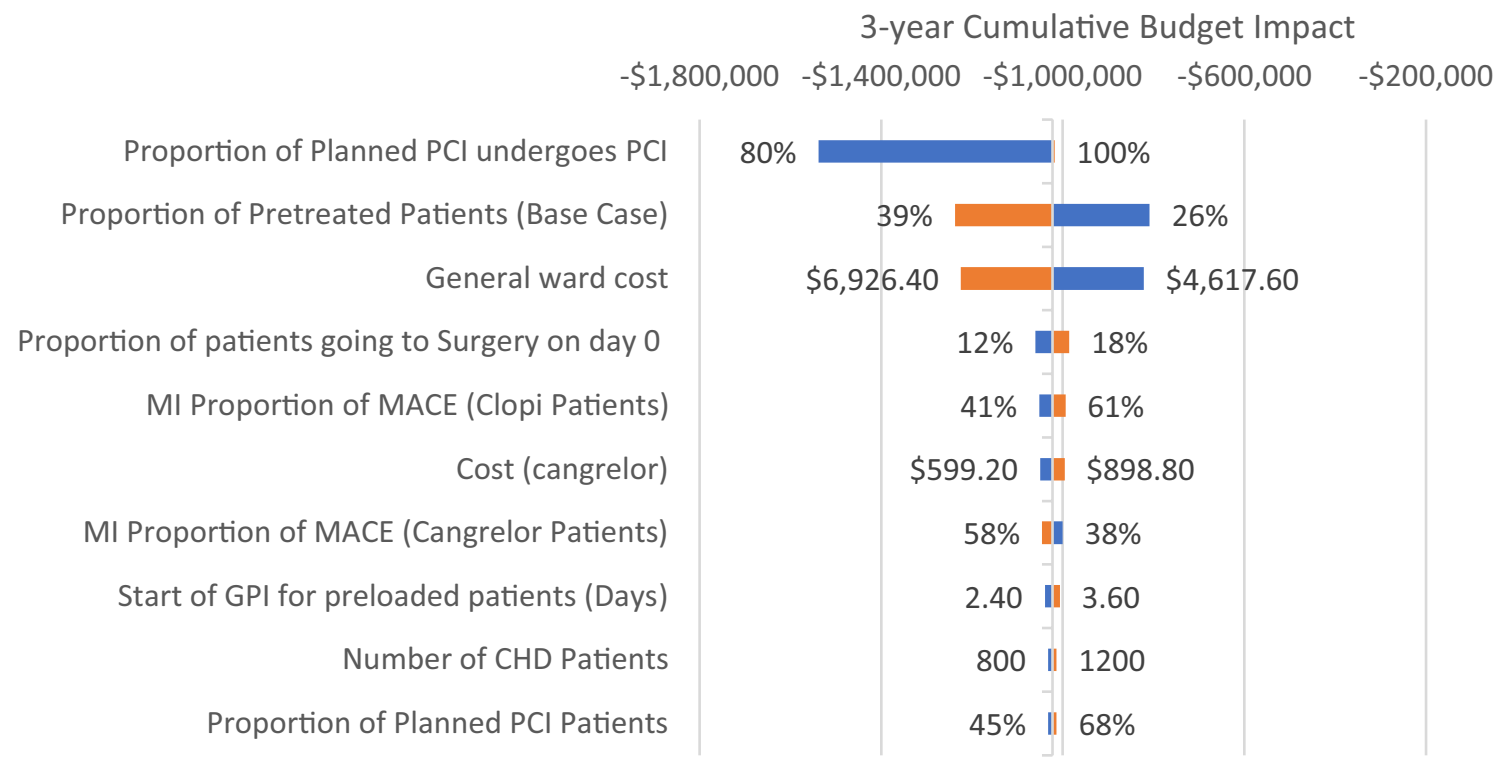

Budget Impact with Low Parameter estimate

Budget Impact with High Parameter Estimate

Fig. 4 Deterministic sensitivity analysis on the 3-year cumulative budget impact (top 10 most influential model parameters). The values on the bars represent the low and high parameter estimate used for the sensitivity analysis. The size of the bar indicates the calculated 3-year cumulative budget impact with the respective low (input reduced

P2 $\mathrm{Y}_{12}$ inhibitors, impacting the model results by $20-50 \%$. General ward costs impacted results by about $19 \%$. All other parameters had a $<5 \%$ impact on model results. Figure 4 illustrates the top 10 inputs with greatest impact on the budget. by $20 \%$ ) or high (input increased by $20 \%$ ) parameter estimate. The base 3-year cumulative budget impact is $-\$ 102,289$. CHD coronary heart disease, Clopi clopidogrel, GPI glycoprotein IIb/IIIa inhibitors, $M A C E$ major adverse cardiovascular event, $M I$ myocardial infarction, $P C I$ percutaneous coronary intervention

\section{Discussion}

The present analysis is the first health economic evaluation developed to estimate the clinical and economic impact of using antiplatelet agents in PCI patients and considering subgroups of increasing angiographic risk. This model 
suggests the budget of a hospital with a constant level of PCI procedures may decline by $13-30 \%$ (assuming a $50-100 \%$ reduction in $\mathrm{P} 2 \mathrm{Y}_{12}$ pretreatment rate) by year 3 if the utilization of cangrelor is increased from $22 \%$ in the base case to $62 \%$ in year 3 in PCI patients with two or more angiographic HRFs.

The cost savings are driven by three main benefits: (1) reduction in ischemic events; (2) reduction in GPI bailout use; and (3) reduced costs for washout of oral $\mathrm{P} 2 \mathrm{Y}_{12}$ inhibitors due to reduced pretreatment. Cost savings from these benefits offset the increase in drug cost and bleeding costs, leading to a decrease in cumulative 3-year costs of $\$ 488,649$ (14.3\%) or $\$ 1,022,891(30 \%)$ for the scenarios of $50 \%$ reduced pretreatment and no pretreatment, respectively.

The CHAMPION PHOENIX angiographic core laboratory analysis indicated improved clinical outcomes with cangrelor for all PCI patients, but greater absolute ischemic benefit was seen in the patients with two or more angiographic HRFs; thus, this was the patient population for which we simulated increased use of cangrelor. This benefit was quantified in the model as a reduction in MACE, translating into cost savings of approximately $5.5 \%$ by year 3 . Patients treated with cangrelor compared with clopidogrel also experienced lower GPI bailout rates, which led to cost savings in GPI bailout of about $2.6 \%$ by year 3 .

Moreover, having cangrelor available for PCI may affect the proportion of CAD patients pretreated with an oral $\mathrm{P} 2 \mathrm{Y}_{12}$ inhibitor. Patients receiving cangrelor would not require pretreatment with an oral $\mathrm{P} 2 \mathrm{Y}_{12}$ inhibitor prior to angiography due to the immediate onset of action of cangrelor. Should these patients require surgery post angiography and remain hospitalized, the rapid offset of cangrelor would not impact scheduling of surgery, and thus be expected to reduce the length of stay prior to surgery. The washout period [2, 8-10] increases the length of stay in these patients, thereby increasing costs (e.g. $>50 \%$ of cangrelor patients were able to have surgery within 1 day post PCI) (Fig. 4). If pretreatment is reduced by $50 \%$ with the use of cangrelor, washout costs by year 3 are expected to be reduced by $\$ 180,818(47.5 \%)$ or $\$ 359,529$ (94\%) with no pretreatment. Similar savings might be realized for hospitals implementing the 2020 NSTEMI ESC guidelines [1].

The use of cangrelor for PCI has been shown to result in a slightly increased incidence of non-severe bleeding compared with clopidogrel, which may lead to higher bleeding event costs [14]. Consistent with published sources, the bleeding events estimated in the model also showed a very slight increase in GUSTO major/moderate bleeding with higher utilization of cangrelor, and therefore bleeding event costs increased only nominally or were similar using the TIMI bleeding definition.

The sensitivity analysis indicated the model was most sensitive to the proportion of patients in whom PCI was planned, which is not surprising since they represent over $90 \%$ of the patients simulated. The model is also sensitive to the inputs relating to the patients requiring washout, such as the proportion of pretreatment and general ward costs that a hospital might incur from pretreating $\mathrm{CABG}$ patients.

These results provide insights about the potential benefits of increasing use of cangrelor among patients with two or more angiographic HRFs. This study also highlights a potential need in stable angina patients. Traditionally, patients with stable angina are perceived to be low-risk as many of these patients receive elective PCI procedures and therefore are typically not considered to be candidates for cangrelor. However, as more than half of the stable angina patients undergoing PCI procedures have two or more angiographic HRFs and are typically managed as outpatients, there may be opportunity for improvement in periprocedural ischemic outcomes, reduction in need for GPI bailout, and shorter duration of washout in this population [18]. Reduction in the need for GPI, whether routine or bailout, may also reduce bleeding, thereby further reducing the cost of acute care [31].

The model has a number of limitations. First, due to the short duration of cangrelor use periprocedurally, cangrelor affects outcomes predominantly in the first 48 hs, whereas the events occurring after the first $48 \mathrm{~h}$ may be attributable to the oral $\mathrm{P}_{2} \mathrm{Y}_{12}$ inhibitor prescribed for secondary prevention of cardiovascular events. However, the early reduction in ischemic events associated with cangrelor use is preserved out to 30 days [14]. In the model, the 48-h clinical outcomes were extrapolated to 30 days as 30-day outcomes are commonly used as quality metrics [32]. The model quantifies the hospitalization costs of washout and bridging, but ignores any harms associated with antiplatelet therapy during washout. However, this simplifying assumption is expected to lead to an underestimate of the total savings in the scenario with lower use of oral $\mathrm{P} 2 \mathrm{Y}_{12}$ pretreatment. This model exclusively evaluated cangrelor and clopidogrel \pm planned GPI use. In contemporary practice, prasugrel or ticagrelor are often used in ACS, but these agents were excluded from the analysis because they have not been extensively studied in the periprocedural setting (i.e. with 48 -h endpoints). Nevertheless, the impact of using the newer oral $\mathrm{P} 2 \mathrm{Y}_{12}$ inhibitors on results of this model should be minimal as the effect of cangrelor is observed within the first few hours [33]. The added cost of these agents during the 30-day period in this model would also be minimal, and events after the 48-h period would be attributable to the oral $\mathrm{P} 2 \mathrm{Y}_{12}$ agent prescribed, which, in this study, is consistent across all patients. Moreover, the washout duration required with these agents is similar or longer than with clopidogrel.

The model also only quantified the cost consequences for CAD patients planned for PCI procedures and patients managed directly with CABG surgery, and excluded patients 
managed medically. Excluding these patients may have underestimated CAD events occurring during the model time horizon. Finally, several methodological assumptions were made to simplify model calculations used in lieu of available clinical trial or real-world data. Sensitivity analyses assessed the top 20 parameters and indicated that some of these assumptions are more impactful to the model results than others. Therefore, the exact impact of these inputs and assumptions is not certain, and further studies are needed to validate the results of this analysis.

\section{Conclusion}

The model presented in this study was used to study the cost consequences of using cangrelor in PCI patients with two or more angiographic HRFs and avoidance of $\mathrm{P}_{2} \mathrm{Y}_{12}$ inhibitor pretreatment in patients managed in hospital with CABG surgery. This analysis suggests having cangrelor available for use in PCI patients with two or more angiographic HRFs may be associated with improved clinical outcomes while providing potential savings to the hospital from fewer CAD events and minimizing $\mathrm{P}_{2} \mathrm{Y}_{12}$ inhibitor washout costs by decreasing pretreatment rates.

Supplementary Information The online version contains supplementary material available at https://doi.org/10.1007/s40256-021-00491-9.

\section{Declarations}

Availability of data and material The assumptions used for the model are all from published sources and referenced throughout the manuscript. The results from the model are reported in the tables. No other data or materials are available from this analysis.

Funding This manuscript was funded by Chiesi, Inc. The authors approved all content and results reported in this manuscript.

Conflict of interest Ivar S. Jensen, Elizabeth Wu, and Philip L. Cyr are employees of PrecisionHEOR, which provides consulting services to the pharmaceutical industry, including Chiesi, Inc. Marc Claussen and Khalid Salahuddin are employees of Chiesi, Inc.; Thomas Winkler was an employee of Chiesi at the time of developing this analysis; and Jayne Prats is a consultant to Chiesi, Inc. Kenneth W. Mahaffey's financial disclosures can be viewed at http://med.stanford.edu/profi les/kenneth-mahaffey. C. Michael Gibson receives research support and consulting fees from Janssen and Johnson \& Johnson, and also receives consulting fees from Bayer. Gabriel Steg discloses the following relationships: research grant from Amarin, Bayer, Sanofi, and Servier, and speaking or consulting fees from Amarin, Amgen, AstraZeneca, Bayer/Janssen, Boehringer-Ingelheim, Bristol-Myers-Squibb, Chiesi, Idorsia, Myokardia, Novartis, Novo-Nordisk, Pfizer, Regeneron, Sanofi, Servier, and The Medicines Company. Gregg W. Stone has received speaker or other honoraria from Cook, Terumo, QOOL Therapeutics, and Orchestra Biomed, and has served as a consultant to Valfix, TherOx, Vascular Dynamics, Robocath, HeartFlow, Gore, Ablative Solutions, Miracor, Neovasc, V-Wave, Abiomed, Ancora, and MAIA. Deepak L. Bhatt discloses the following relationships: Advi- sory Board: Cardax, CellProthera, Cereno Scientific, Elsevier Practice Update Cardiology, Janssen, Level Ex, Medscape Cardiology, MyoKardia, NirvaMed, Novo Nordisk, PhaseBio, PLx Pharma, and Regado Biosciences; Board of Directors: Boston VA Research Institute, Society of Cardiovascular Patient Care, and TobeSoft; Chair: American Heart Association Quality Oversight Committee; Data Monitoring Committees: Baim Institute for Clinical Research (formerly Harvard Clinical Research Institute, for the PORTICO trial, funded by St. Jude Medical, now Abbott), Cleveland Clinic (including for the ExCEED trial, funded by Edwards), Contego Medical (Chair, PERFORMANCE 2), Duke Clinical Research Institute, Mayo Clinic, Mount Sinai School of Medicine (for the ENVISAGE trial, funded by Daiichi Sankyo), and Population Health Research Institute; Honoraria: American College of Cardiology (Senior Associate Editor, Clinical Trials and News, ACC.org; Chair, ACC Accreditation Committee), Baim Institute for Clinical Research (formerly Harvard Clinical Research Institute; REDUAL PCI Clinical Trial Steering Committee funded by Boehringer Ingelheim; AEGIS-II executive committee funded by CSL Behring), Belvoir Publications (Editor in Chief, Harvard Heart Letter), Canadian Medical and Surgical Knowledge Translation Research Group (Clinical Trial Steering Committees), Duke Clinical Research Institute (Clinical Trial Steering Committees, including for the PRONOUNCE trial, funded by Ferring Pharmaceuticals), HMP Global (Editor in Chief, Journal of Invasive Cardiology), Journal of the American College of Cardiology (Guest Editor, Associate Editor), K2P (Co-Chair, interdisciplinary curriculum), Level Ex, Medtelligence/ReachMD (CME steering committees), MJH Life Sciences, Population Health Research Institute (for the COMPASS Operations Committee, Publications Committee, Steering Committee, and USA national co-leader, funded by Bayer), Slack Publications (Chief Medical Editor, Cardiology Today's Intervention), Society of Cardiovascular Patient Care (Secretary/ Treasurer), WebMD (CME Steering Committees); Other: Clinical Cardiology (Deputy Editor), NCDR-ACTION Registry Steering Committee (Chair), VA CART Research and Publications Committee (Chair); Research Funding: Abbott, Afimmune, Amarin, Amgen, AstraZeneca, Bayer, Boehringer Ingelheim, Bristol-Myers Squibb, Cardax, CellProthera, Cereno Scientific, Chiesi, CSL Behring, Eisai, Ethicon, Ferring Pharmaceuticals, Forest Laboratories, Fractyl, Garmin, HLS Therapeutics, Idorsia, Ironwood, Ischemix, Janssen, Lexicon, Lilly, Medtronic, MyoKardia, NirvaMed, Novartis, Novo Nordisk, Owkin, Pfizer, PhaseBio, PLx Pharma, Regeneron, Roche, Sanofi, Synaptic, The Medicines Company, and 89Bio; Royalties: Elsevier (Editor, Cardiovascular Intervention: A Companion to Braunwald's Heart Disease); Site Co-Investigator: Abbott, Biotronik, Boston Scientific, CSI, St. Jude Medical (now Abbott), Svelte; Trustee: American College of Cardiology; and Unfunded Research: FlowCo, Merck, Takeda.

Ethics approval Not applicable.

Consent to participate Not applicable.

Consent for publication Not applicable.

Code availability Not applicable.

Author contributions ISJ, EW: Conceptualization, methodology, model programming, writing, review, and editing. PLC, MC, TW, KS, JP: Conceptualization, methodology, validation, writing, review and editing. KWM, CMG, GS, GWS, and DLB: Validation, writing, review, and editing.

Open Access This article is licensed under a Creative Commons Attribution-NonCommercial 4.0 International License, which permits any non-commercial use, sharing, adaptation, distribution and reproduction in any medium or format, as long as you give appropriate credit to the 
original author(s) and the source, provide a link to the Creative Commons licence, and indicate if changes were made. The images or other third party material in this article are included in the article's Creative Commons licence, unless indicated otherwise in a credit line to the material. If material is not included in the article's Creative Commons licence and your intended use is not permitted by statutory regulation or exceeds the permitted use, you will need to obtain permission directly from the copyright holder. To view a copy of this licence, visit http://creativecommons.org/licenses/by-nc/4.0/.

\section{References}

1. Collet JP, Thiele H, Barbato E, Barthelemy O, Bauersachs J, Bhatt DL, et al. 2020 ESC Guidelines for the management of acute coronary syndromes in patients presenting without persistent STsegment elevation. Eur Heart J. 2021;42(14):1289-367.

2. Levine GN, Bates ER, Blankenship JC, Bailey SR, Bittl JA, Cercek B. 2011 ACCF/AHA/SCAI Guideline for Percutaneous Coronary Intervention. A report of the American College of Cardiology Foundation/American Heart Association Task Force on Practice Guidelines and the Society for Cardiovascular Angiography and Interventions. J Am Coll Cardiol. 2011;58(24):e44-122.

3. Johnson TW, Mumford AD, Scott LJ, Mundell S, Butler M, Strange JW. A Study of Platelet Inhibition, Using a 'Point of Care' Platelet Function Test, following Primary Percutaneous Coronary Intervention for ST-Elevation Myocardial Infarction [PINPOINTPPCI]. PLoS ONE. 2015;10(12):e0144984.

4. Kubica J, Adamski P, Ostrowska M, Sikora J, Kubica JM, Sroka WD. Morphine delays and attenuates ticagrelor exposure and action in patients with myocardial infarction: the randomized, double-blind, placebo-controlled IMPRESSION trial. Eur Heart J. 2016;37(3):245-52

5. Silvain J, Storey RF, Cayla G, Esteve JB, Dillinger JG, Rousseau H. P2Y12 receptor inhibition and effect of morphine in patients undergoing primary PCI for ST-segment elevation myocardial infarction. The PRIVATE-ATLANTIC study. Thromb Haemost. 2016;116(2):369-78.

6. McEvoy JW, Ibrahim K, Kickler TS, Clarke WA, Hasan RK, Czarny MJ, et al. Effect of intravenous fentanyl on ticagrelor absorption and platelet inhibition among patients undergoing percutaneous coronary intervention. Circulation. 2018;137(3):307-9.

7. Goli RR, Ibrahim K, Shah R, Kickler TS, Clarke WA, Resar JR, et al. The dose and timing of fentanyl impacts on ticagrelor absorption and platelet inhibition during percutaneous coronary intervention: the PACIFY randomized clinical trial. J Invasive Cardiol. 2019;31(9):265-71

8. PLAVIX (clopidogrel bisulfate) tablets, for oral use. 1997 May 2019. https://www.accessdata.fda.gov/drugsatfda_docs/label/ 2018/020839s070lbl.pdf. Accessed 30 July 2019

9. EFFIENT (prasugrel) tablets. 2009 September 2011. https://www. accessdata.fda.gov/drugsatfda_docs/label/2011/022307s003lbl. pdf. Accessed 30 July 2019

10. BRILINTA (ticagrelor) tablets, for oral use. 2011 September 2016. https://www.accessdata.fda.gov/drugsatfda_docs/label/ 2016/022433s0201bl.pdf. Accessed 30 July 2019

11. Bhatt DL, Stone GW, Mahaffey KW, Gibson CM, Steg PG, Hamm $\mathrm{CW}$. Effect of platelet inhibition with cangrelor during PCI on ischemic events. N Engl J Med. 2013;368(14):1303-13.

12. Bhatt DL, Lincoff AM, Gibson CM, Stone GW, McNulty S, Montalescot G. Intravenous platelet blockade with cangrelor during PCI. N Engl J Med. 2009;361(24):2330-41.
13. Harrington RA, Stone GW, McNulty S, White HD, Lincoff AM, Gibson CM. Platelet inhibition with cangrelor in patients undergoing PCI. N Engl J Med. 2009;361(24):2318-29.

14. Steg PG, Bhatt DL, Hamm CW, Stone GW, Gibson CM, Mahaffey $\mathrm{KW}$. Effect of cangrelor on periprocedural outcomes in percutaneous coronary interventions: a pooled analysis of patient-level data. Lancet. 2013;382(9909):1981-92.

15. Vaduganathan M, Harrington RA, Stone GW, Steg PG, Gibson $\mathrm{CM}$, Hamm CW, et al. Variation in patient profiles and outcomes in US and non-US subgroups of the cangrelor versus standard therapy to achieve optimal management of platelet inhibition (CHAMPION) PHOENIX trial. Circ Cardiovasc Interv. 2016;9(6):e003612-e.

16. Ibanez B, James S, Agewall S, Antunes MJ, Bucciarelli-Ducci C, Bueno H, et al. 2017 ESC Guidelines for the management of acute myocardial infarction in patients presenting with ST-segment elevation: the Task Force for the management of acute myocardial infarction in patients presenting with ST-segment elevation of the European Society of Cardiology (ESC). Eur Heart J. 2018;39(2):119-77.

17. Neumann FJ, Sousa-Uva M, Ahlsson A, Alfonso F, Banning AP, Benedetto U, et al. 2018 ESC/EACTS Guidelines on myocardial revascularization. Eur Heart J. 2019;40(2):87-165.

18. Stone GW, Genereux P, Harrington RA, White HD, Gibson CM, Steg PG, et al. Impact of lesion complexity on peri-procedural adverse events and the benefit of potent intravenous platelet adenosine diphosphate receptor inhibition after percutaneous coronary intervention: core laboratory analysis from 10854 patients from the CHAMPION PHOENIX trial. Eur Heart J. 2018;39(46):4112-21.

19. Cavender M, Harrington R, Stone G, Deliargyris E, Steg P, Gibson C. Ischemic events occur early in patients undergoing PCI and are reduced with cangrelor: findings from CHAMPION PHOENIX. J Am Coll Cardiol. 2017;69(11 Suppl):25.

20. Cavender MA, Bhatt DL, Stone GW, White HD, Steg PG, Gibson $\mathrm{CM}$, et al. Consistent reduction in periprocedural myocardial infarction with cangrelor as assessed by multiple definitions: findings from CHAMPION PHOENIX (cangrelor versus standard therapy to achieve optimal management of platelet inhibition). Circulation. 2016;134(10):723-33.

21. Jatene T, Harrington RA, Stone GW, Steg PG, Gibson CM, Hamm $\mathrm{CW}$, et al. Investigator-reported bleeding versus post hoc adjudication of bleeding: lessons from the CHAMPION PHOENIX trial. J Am Coll Cardiol. 2016;67(5):596-8.

22. Qamar A, Bhatt DL. Optimizing the use of cangrelor in the real world. Am J Cardiovasc Drugs. 2017;17(1):5-16.

23. Stone GW, McLaurin BT, Cox DA, Bertrand ME, Lincoff AM, Moses JW. Bivalirudin for patients with acute coronary syndromes. N Engl J Med. 2006;355(21):2203-16.

24. Badri M, Abdelbaky A, Li S, Chiswell K, Wang TY. Precatheterization use of P2Y12 inhibitors in non-ST-elevation myocardial infarction patients undergoing early cardiac catheterization and in-hospital coronary artery bypass grafting: insights from the National Cardiovascular Data Registry ${ }^{\circledR}$. J Am Heart Assoc. 2017;6(9):e006508.

25. Vaduganathan M, Harrington RA, Stone GW, Deliargyris EN, Steg PG, Gibson CM. Evaluation of ischemic and bleeding risks associated with 2 parenteral antiplatelet strategies comparing cangrelor with glycoprotein IIb/IIIa inhibitors: an exploratory analysis from the CHAMPION trials. JAMA Cardiol. 2017;2(2):127-35.

26. Mehran R, Rao SV, Bhatt DL, Gibson CM, Caixeta A, Eikelboom J, et al. Standardized bleeding definitions for cardiovascular clinical trials: a consensus report from the Bleeding Academic Research Consortium. Circulation. 2011;123(23):2736-47.

27. Ben Morrison T, Horst BM, Brown MJ, Bell MR, Daniels PR. Bridging with glycoprotein IIb/IIIa inhibitors for periprocedural 
management of antiplatelet therapy in patients with drug eluting stents. Catheter Cardiovasc Interv. 2012;79(4):575-82.

28. Dressler R, Dryer MM, Coletti C, Mahoney D, Doorey AJ. Altering overuse of cardiac telemetry in non-intensive care unit settings by hardwiring the use of American Heart Association guidelines. JAMA Intern Med. 2014;174(11):1852-4.

29. Bureau of Labor Statistics. Medical Care Consumer Price Index. 2018. http://www.bls.gov/data/. Accessed 30 Aug 2019

30. AnalySource Database. 2019. https://www.analysource.com/. Accessed 30 Oct 2019
31. Vaduganathan M, Harrington RA, Stone GW, Deliargyris EN, Steg PG, Gibson CM, et al. Cangrelor with and without glycoprotein IIb/IIIa inhibitors in patients undergoing percutaneous coronary intervention. J Am Coll Cardiol. 2017;69(2):176-85.

32. US Centers for Medicare \& Medicaid Services. Outcome Measures. 2018. https://www.cms.gov/Medicare/Quality-InitiativesPatient-Assessment-Instruments/HospitalQualityInits/Outco meMeasures.html. Accessed 5 Sep 2019

33. Franchi F, Rollini F, Rivas A, Wali M, Briceno M, Agarwal M, et al. Platelet inhibition with cangrelor and crushed ticagrelor in patients with ST-s. Circulation. 2019;139(14):1661-70.

\section{Authors and Affiliations}

\section{Ivar S. Jensen ${ }^{1}$ (1) Elizabeth Wu ${ }^{1} \cdot$ Philip L. Cyr $^{1,2} \cdot$ Marc Claussen $^{3} \cdot$ Thomas Winkler $^{3} \cdot$ Khalid Salahuddin $^{3}$. Jayne Prats ${ }^{4} \cdot$ Kenneth W. Mahaffey $^{5} \cdot$ Charles Michael Gibson $^{6} \cdot$ Philippe Gabriel Steg $^{7} \cdot$ Gregg W. Stone $^{8}$. Deepak L. Bhatt ${ }^{9}$}

1 Precision Health Economics, 133 Federal Street, 10th floor, Boston, MA 02110, USA

2 University of North Carolina at Charlotte, College of Health and Human Services, Charlotte, NC, USA

3 Chiesi, Inc., Cary, NC, USA

4 Elysis, Carlisle, MA, USA

5 Department of Medicine, Stanford Center for Clinical Research, Stanford, CA, USA

6 Division of Cardiology, Beth Israel Deaconess Medical Center, Boston, MA, USA
7 Université de Paris, Assistance Publique-Hôpitaux de Paris, Hôpital Bichat, INSERM U-1148, Paris, France

8 The Zena and Michael A. Wiener Cardiovascular Institute, Icahn School of Medicine at Mount Sinai, and the Cardiovascular Research Foundation, New York, NY, USA

9 Brigham and Women's Hospital Heart and Vascular Center, and Harvard Medical School, Boston, MA, USA 